\title{
Evaluation of Microbiome-Host Relationships in the Zebrafish Gastrointestinal System Reveals Adaptive Immunity Is a Target of Bis(2-ethylhexyl) Phthalate (DEHP) Exposure
}

Ondrej Adamovsky, Amanda N. Buerger, Hana Vespalcova, Shahadur R. Sohag, Amy T. Hanlon, Pamela E. Ginn, Serena L. Craft, Stanislav Smatana, Eva Budinska, Maria Persico, Joseph H. Bisesi, Jr., * and Christopher J. Martyniuk*

Cite This: Environ. Sci. Technol. 2020, 54, 5719-5728

Read Online

\section{ACCESS I}

WIII Metrics \& More

Article Recommendations

Supporting Information

ABSTRACT: To improve physical characteristics of plastics such as flexibility and durability, producers enrich materials with phthalates such as di-2-(ethylhexyl) phthalate (DEHP). DEHP is a high production volume chemical associated with metabolic and immune disruption in animals and humans. To reveal mechanisms implicated in phthalate-related disruption in the gastrointestinal system, male and female zebrafish were fed DEHP (3 ppm) daily for two months. At the transcriptome level, DEHP significantly upregulated gene networks in the intestine associated with helper $\mathrm{T}$ cells' (Th1, Th2, and Th17) specific pathways. The activation of gene networks associated with adaptive immunity was linked to the suppression of networks for tight junction, gap junctional intercellular communication, and transmembrane transporters, all

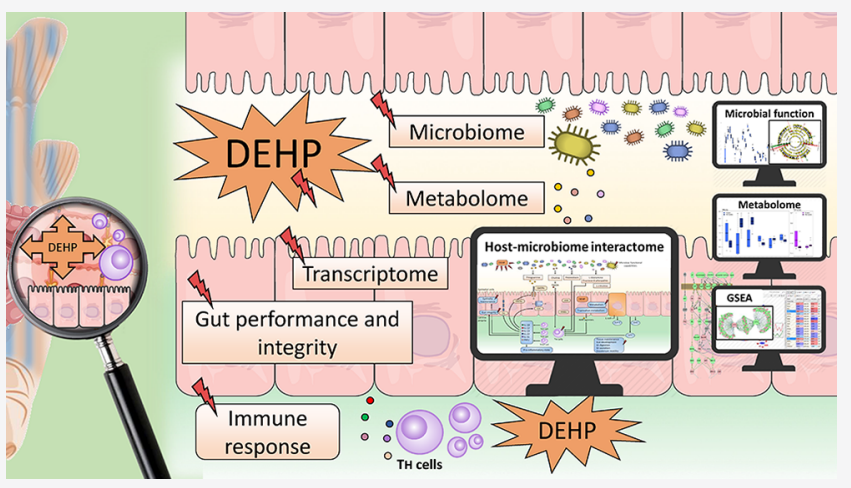
of which are precursors for impaired gut integrity and performance. On a class level, DEHP exposure increased Bacteroidia and Gammaproteobacteria and decreased Verrucomicrobiae in both the male and female gastrointestinal system. Further, in males there was a relative increase in Fusobacteriia and Betaproteobacteria and a relative decrease in Saccharibacteria. Predictive algorithms revealed that the functional shift in the microbiome community, and the metabolites they produce, act to modulate intestinal adaptive immunity. This finding suggests that the gut microbiota may contribute to the adverse effects of DEHP on the host by altering metabolites sensed by both intestinal and immune Th cells. Our results suggest that the microbiome-gut-immune axis can be modified by DEHP and emphasize the value of multiomics approaches to study microbiome-host interactions following chemical perturbations.

\section{INTRODUCTION}

Phthalates are widely used as softeners in plastic materials (e.g., polyvinyl chloride; PVC), food packaging, cosmetics, children's toys, medical products (blood bags, tubing) and other industrial products. One of the most widely produced phthalates is di-2-ethylhexyl phthalate (DEHP), which accounted for approximately $40 \%$ of world consumption of plasticizers in 2014. ${ }^{1}$ Similar to other phthalates, DEHP is not covalently bound to the plastic polymer. This enables leaching, resulting in DEHP becoming pseudopersistent in the environment and within the body. Significant leaching and volatilization of phthalates from parental material leads to ubiquitous exposures in human populations ${ }^{2}$ and environments. ${ }^{3}$ In fact, metabolite biomarkers of eight major phthalates have been detected in urine in $89 \%$ to $98 \%$ of the United States (US) population. ${ }^{4}$ While there are many routes of exposure (occupational, direct contact with plastics), diet remains one of the dominant pathways for contact with phthalates such as DEHP. ${ }^{5}$ A recent review showed that humans are chronically exposed to phthalates, as the majority of foods (from vegetables to meat) contain phthalates, dominated by DEHP in the parts per million concentration range. ${ }^{6,7}$ Moreover, phthalates are intentionally used as film coating agents in orally ingested tablets and in numerous types of modified release drug delivery systems, such as entericcoated and delayed-release tablets and enteric-coated capsules. ${ }^{8}$ Thus, DEHP comes into direct contact with the gastrointestinal system of the host and its microbiome. Nevertheless, the effects of DEHP specifically on the complex

Received: January 31, 2020

Revised: April 6, 2020

Accepted: April 7, 2020

Published: April 7, 2020 
microbiome-gut axis and health outcomes are unknown and warrant further investigation.

Epidemiological evidence suggests that over a dozen phthalates present in the environment (food, water, air), and those derived from household products, exacerbate human disease with exposure. Phthalates are associated with a higher incidence of specific cancers (breast and skin cancer), obesityrelated pathology (insulin resistance and type II diabetes), and neurological conditions (attention-deficit hyperactivity disorder) among other diseases. ${ }^{9}$ DEHP in particular is linked to metabolic disruption and obesity in children ${ }^{10,11}$ and adults, ${ }^{12,13}$ and more studies associate phthalates with the impact on adaptive immune system ${ }^{14}$ and connected adverse health outcomes, e.g., asthma and allergies. ${ }^{15,16}$ The effect of DEHP on adaptive immunity, specifically Th cells, is corroborated by findings in in vitro and in vivo models that show that DEHP promotes Th1 and Th17 differentiation and immune responses characterized by nonphysiological synthesis of a spectrum of Th cytokines (e.g., Il-4, Il-21, Il-13). ${ }^{14,17,18}$ Studies focused on understanding the effects of xenobiotics on the host immune system reveal the importance of the host microbiome-intestinal immune axis; in addition to a direct effect, changes in microbial core functions may be responsible for inflammatory and metabolic disorders. ${ }^{19}$ Accumulating evidence indicates that the effect of xenobiotics on gut microbiome composition and diversity plays a role in mediating chemical toxicity and may exacerbate human diseases. ${ }^{20}$ A recent study using a rodent model showed the effect of low-dose exposure to diethyl phthalate (DEP) on composition of gut microbiome, specifically on phyla Firmicutes and Bacteroidetes; ${ }^{21}$ however there is a lack of holistic investigations into the impact of phthalates on a complex microbiome-gut-immune axis.

Moreover, data are sparse on how environmental chemicals, such as phthalates, affect the microbiome-gut axis, and there are fundamental gaps in our understanding of the precise mechanisms by which chemical-induced microbial shifts affect the immune system, especially for low, subchronic chemical exposures. The objective of this study was to determine the mechanisms by which phthalates impact the gastrointestinal system and potentially host immunity, with emphasis on the interaction with the host microbiome. Zebrafish, a widely used toxicological and human health model, were fed an environmentally relevant concentration of DEHP (3 ppm) in feed for 2 months. The concentration of DEHP used in this study is within the range of concentrations of DEHP found in food, specifically with higher levels of fat (tens of $\mathrm{mg} / \mathrm{kg}(\mathrm{ppm}))^{6}$ and in waste waters. ${ }^{22}$ To determine the impact of DEHP on microbiome-gut-immune networks, we analyzed the host gastrointestinal transcriptome and established a workflow to analyze the effect on host microbiome community. Last, using computational approaches, we predict functional changes in bioactive microbial metabolites related to DEHP exposure. We hypothesized that a subchronic 2 month exposure to DEHP would alter the expression of gene networks related to metabolism and the adaptive immune systems. These data generate novel insight into plasticizer exposure from both an environmental as well as a human health perspective.

\section{METHODOLOGY}

Experimental Design. All experiments were conducted under the supervision and approval of the University of Florida Institutional Animal Care and Use Committee (IACUC no.201708217). Zebrafish (Danio rerio) at $\sim 8$ months of age were divided into 20 tanks (10 replicate tanks/treatment). Each tank contained three males and three females. Tanks were randomly assigned one of two treatments: control (normal food) or DEHP ( $3 \mathrm{mg} / \mathrm{kg}$ DEHP in food). Methods for preparation of the diet were previously reported in Buerger et al. ${ }^{23}$ Briefly, food was prepared by mixing DEHP (CAS 117 81-7, Sigma-Aldrich, cat. no. 36735 ) with $100 \%$ menhaden oil, followed by slow addition to Adult Zebrafish Diet (Zeigler, Gardner, PA) in a food mixer. Control food was mixed with menhaden oil alone. Animals were fed $10 \mathrm{mg}$ of their respective food per day for 8 weeks. The experiment was performed under stable conditions with water temperatures of $26 \pm 1{ }^{\circ} \mathrm{C}$ and a $14 / 10 \mathrm{~h} \mathrm{light/dark} \mathrm{cycle.} \mathrm{Fish} \mathrm{length} \mathrm{and}$ weight were measured biweekly to assess weight gain and calculate body mass index (BMI). After the exposure, the fish were anesthetized and then euthanized with an overdose $(250$ $\mathrm{mg} / \mathrm{L}$ ) of buffered MS222 (ethyl 3-aminobenzoate methanesulfonate) and immediately dissected. Gastrointestinal tissue (GI) and liver samples were placed on Davidson's fixative for histopathological evaluation. Tissue samples (GI, liver, and fecal content) were also flash frozen in liquid nitrogen and stored at $-80{ }^{\circ} \mathrm{C}$.

The Analysis of DEHP Content in the Feed. Concentrations of DEHP in the food were measured following methods previously reported in Buerger et al. ${ }^{23}$ Briefly, three subsamples of the bulk food were extracted in 100\% Optima grade acetonitrile (Fisher Scientific, Hampton, NH, USA cat. no.: A996-1). A subsample of the extract was then analyzed using an Agilent $7890 \mathrm{~B}$ gas chromatograph coupled with a $7000 \mathrm{C}$ triple quadrupole mass spectrometer. Results from the three food subsamples were averaged and the standard deviation calculated.

Transcriptomic Analysis of the Gastrointestinal Tissue. The gastrointestinal RNA was extracted using a hand-held homogenizer (Ika T10, Germany) and was processed using TRIzol Reagent (Thermo Fisher Scientific, USA), as per the manufacturer's procedure $(N=10$ individuals per treatment, each collected from separate replicate tanks within the study). Nucleic acid pellets were reconstituted in Milli-Q water. Extracted RNA was cleaned and DNase-treated using Qiagen RNeasy Mini Kit (Qiagen) as per the manufacturer's protocol. Samples were assessed for quantity and quality using a ThermoFisher Qubit 3.0 (RNA high sensitivity assay kit, Q32852) and the Agilent 2100 Bioanalyzer (Agilent, USA). Those samples with the highest quality were used for microarray analysis. The mean RNA Integrity Number (RIN) for samples was 7.9 ( $\min -\max 7.2-9.1)$. Microarray processing was performed according to the manufacturer's protocols (Agilent LowInput QuickAmp Labeling Kit (\#51902331) and laboratory protocols $(N=6-8$ per group) $) .{ }^{24}$ The Agilent Zebrafish platform $(4 \mathrm{x} 44 \mathrm{~K}, \mathrm{~V} 3)$ was used to analyze the GI transcriptome, and $17 \mathrm{~h}$ hybridization was performed (Agilent hybridization kit (\#5188-5242)) according to the manufacturer's protocol. Microarray slides were scanned using an Agilent DNA Microarray Scanner with Surescan highresolution technologies and raw data extracted by Agilent Feature Extraction Software. Raw transcriptomic data are publicly available at NCBI Gene Expression Omnibus (accession no. GSE136285). JMP Genomics (v8) were used to process the raw data, normalize the intensities ( 1.8 value as limit of detection of intensity), and perform differentially expressed genes analysis (DEGs). Differentially expressed 
genes were those with a false discovery rate $($ FDR $)<0.05$. A total number of 26,482 genes (out of 43813 ) were successfully mapped to human homologues using the Name + Alias function in Pathway Studio (v10). For Gene Set Enrichment Analysis (GSEA), genes were permutated 1000 times using the Kolmogorov-Smirnov classic approach as an enrichment algorithm. To broaden the analysis, all pathways were expanded to include cell processes and functional classes in target gene seeds. The enrichment $p$-value cutoff was set at $p<$ 0.05. Sub-network enrichment analysis (SNEA) was also performed to determine the specific gene subnetworks affected by DEHP. The enrichment $\mathrm{p}$-value for gene seeds was set at $p$ $<0.05$.

Selected differentially expressed genes with FDR $<0.05$ were confirmed by quantitative PCR (qPCR; Figure S1). The samples used for qPCR were identical to those used for microarrays. To perform qPCR, $1 \mu \mathrm{g}$ of column purified RNA ( $N=6-8$ individuals from different tanks per group) was converted to cDNA using iScript (BioRad, USA) and the T100 Thermal Cycler (BioRad, USA). We followed the best practice according to Buerger at al. ${ }^{23}$ that includes all standard controls (No Reverse Transcriptase (NRT) and No Treatment Controls (NTC)).

Real-time PCR (qPCR) was performed using the CFX Connect Real-Time PCR Detection System (BioRad, USA) with SsoFast EvaGreen Supermix (BioRad, Hercules, CA, USA). Primer sets for target genes were collected from the literature or designed with Primer 3, and the cycling parameters were initiated at $95{ }^{\circ} \mathrm{C}(30 \mathrm{~s})$ followed by 39 cycles of $95{ }^{\circ} \mathrm{C}(5 \mathrm{~s})$ and $55^{\circ} \mathrm{C}(7 \mathrm{~s})$. The dissociation melt curve was generated, starting at 65.0 and ending at $95.0{ }^{\circ} \mathrm{C}$, with increments of $0.5{ }^{\circ} \mathrm{C}$ every $5 \mathrm{~s}$. Each primer set was tested for linearity and efficiency generated by a dilution series from a cDNA pool of zebrafish GI tissue. Two reference genes (rib12 and $b 2 \mathrm{~m}$ ) were assessed for normalization using CFX Manager software and used for normalization (M stability value of 0.91 , $\mathrm{CV}=0.34)$ after being deemed appropriate. Normalized gene expression was extracted using CFX Manager with the relative $\Delta \Delta \mathrm{Cq}$ method (baseline subtracted). The results were statistically evaluated using Kruskal-Wallis nonparametric ANOVA test performed using GraphPad Prism 5.0.

Fecal Sample Preparation and $16 \mathrm{~S}$ Sequencing. Fecal samples $(N=10-13$ individuals were measured, with at least one fish collected from each tank replicate) were extracted using the Zymo Quick-DNA Fecal/Soil Microbe Miniprep Kit (D6010) using beads for sample homogenization. Extracted DNA was quantified using the Qubit ThermoFisher and the dsDNA HS Assay Kit (Q32851/Q32854). Following this, samples were adjusted to $5 \mathrm{ng} / \mu \mathrm{L}$. The AccuPrime PCR protocol was used to amplify the $16 \mathrm{~S}$ (V3 and V4) region using AccuPrime Taq DNA Polymerase (ThermoFisher Scientific Cat. No: 12346-086) according to the manufacturer's instruction. The amplicon size was verified by agarose gel electrophoresis for the correct amplicon size ( 500-600 base pairs). The PCR amplicon was purified by magnetic beads AMPure XP Beads (A63880). We indexed the samples by Nextera XT DNA Library Preparation Kit (FC-131-2004) with AccuPrime Taq DNA Polymerase, High Fidelity (12346-086) and Nextera XT Index primers (S513-S522, N716-N729) according to the manufacturer protocol. The PCR product was purified by magnetic beads: AMPure XP Beads (A63880). Each indexed sample was quantified using the Qubit Fluorometer and diluted to a final concentration $4 \mathrm{nM}$ using
$10 \mathrm{mM}$ Tris $(\mathrm{pH} 8.5)$. The 91 samples were combined $(5 \mu \mathrm{L} /$ sample), and the pooled library was quantified using a NEBNext NGS Library Quant Kit for Illumina (New England Bio, Cat. No.: E7630S) and sequenced on an Illumina MiSeq (run id. 180315 M01105 $0019000000000-B K N P 7)$ in the Interdisciplinary Center for Biotechnology Research, University of Florida according to established protocols.

Analysis of 16s Sequencing Data. 16s rRNA sequences were first subjected to length filtering and quality filtering using the Trimmomatic software package. ${ }^{25}$ Following the quality filtering, closed OTU picking was performed using the Uclust algorithm with a $97 \%$ sequence similarity threshold. ${ }^{26}$ Obtained representative sequences were assigned taxonomy by a Uclust search with a consensus (threshold 0.51, n-hits 3 ). Both OTU picking and taxonomy assignments were executed using the Quantitative Insights into Molecular Ecology $(\text { QIIME })^{27}$ framework and used Silva $(v 123)^{28}$ as the $16 \mathrm{~s}$ rRNA reference database. As the final step, abundances of Kyoto Encyclopedia of Genes and Genomes (KEGG) ${ }^{29}$ orthologs for each sample were predicted using the PICRUSt model (Phylogenetic Investigation of Communities by Reconstruction of Unobserved States) ${ }^{30}$ tool retrained for the Silva database. Resulting abundance profiles were summarized according to the KEGG BRITE Metabolism hierarchy.

Bacterial Metabolome Predictions. The outcome of the bacterial functional profile (PICRUSt) was used to predict the shift in bacterial metabolome. The pipeline was thus expanded by a normalization step based on the algorithm MUSICC ${ }^{31}$ to rescale the $\mathrm{KO}$ abundances to average gene copy number, with the final aim to correct the predicted KO counts derived from the original OTU table. Further, we adapted and reimplemented the PRMT (Predicted Relative Metabolomic Turnover $)^{32}$ and the MIMOSA ${ }^{33}$ approaches to produce microbial community-wide metabolic potential scores (CMP or PRMT scores) for each metabolite annotated in the knowledge database KEGG. The output of the applied workflow is estimated community-wide metabolic potential scores for each metabolite per metagenomics sample.

Statistical Analysis of 16s Data. All metagenomic and metabolomic data (QIIME taxonomy assignment, PICRUSt metabolic pathways and MIMOSA estimated metabolome) were analyzed for each gender separately. Only taxa with at least $1 \%$ relative abundance in at least one sample were kept for the analysis. The PICRUSt KO terms had to pass the $0.1 \%$ relative abundance threshold. The metabolites were considered for analysis if at least one of the group medians (control or DEHP) exceeded the value of 0.1 .

Both QIIME taxonomy estimates and PICRUST metabolic pathway estimates data were transformed using centered logratio transformation, ${ }^{34}$ and a two-way unpaired $t$ test was used for comparison of the differences between the groups, for each taxa or KO term separately.

The estimated metabolome was tested by a nonparametric Mann-Whitney $U$ test. All resulting $p$ values were adjusted for multiple hypothesis testing using the Benjamini-Hochberg procedure with a significance threshold of 0.1 . The statistical analyses were performed in $\mathrm{R}$ version $3.4 .4 .^{35}$

Analysis of the Glucose and Glycogen in Liver. Hepatic glycogen levels were measured in duplicate using the Colorimetric Assay kit (BioVision, no. K646-100) according the manufacturer's protocol. A glucose background level was determined by repeating the protocol without the addition of 


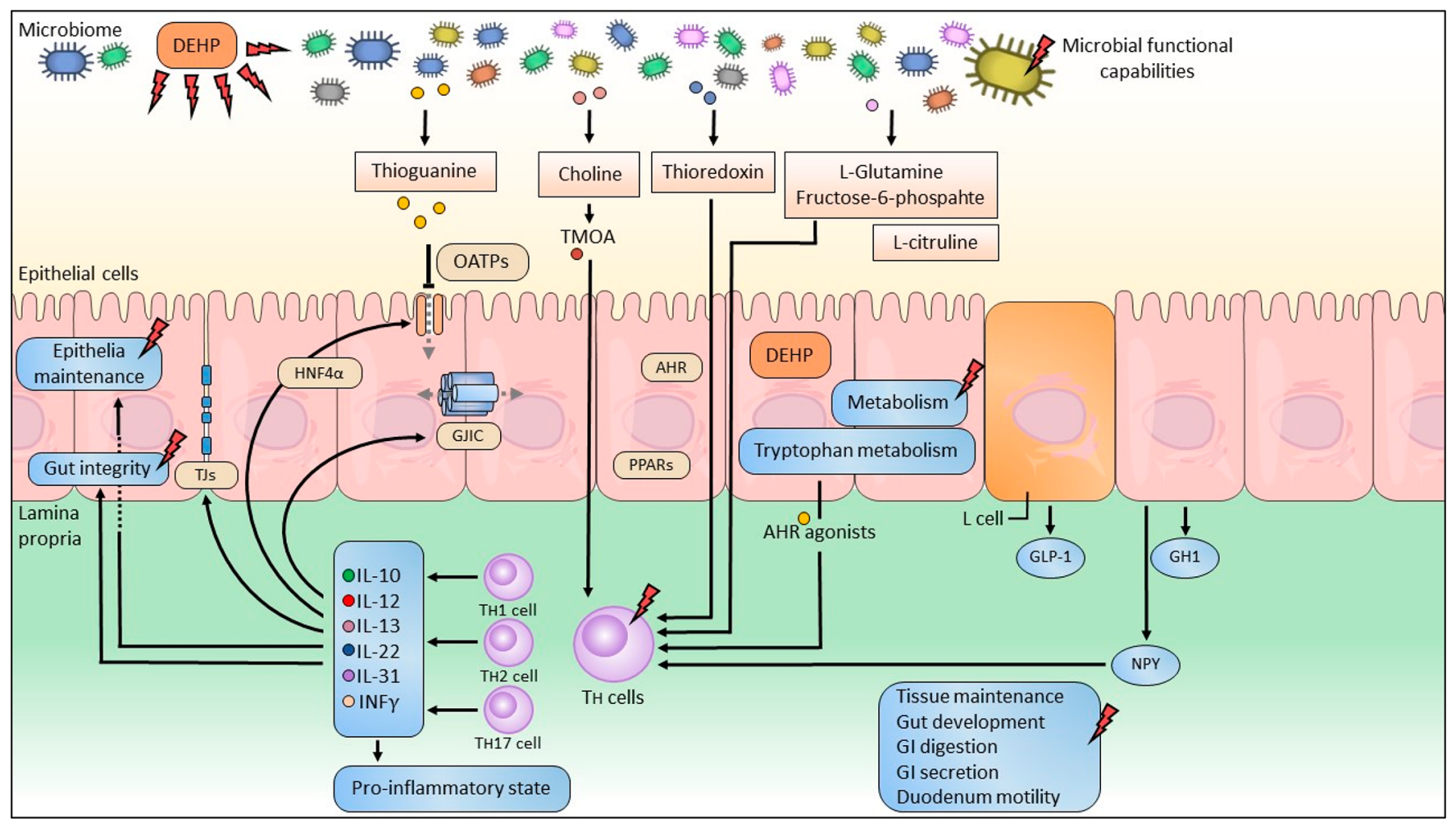

Figure 1. Proposed DEHP interaction with the host's microbiome-gastrointestinal-immune system. DEHP was identified to affect both the gut microbial community and spectrum of biological processes in the gut tissue. DEHP significantly upregulated gene networks associated with helper T cells' (Th1, Th2, Th17) specific pathways that, in addition to direct effect of DEHP, may alter the gut integrity and performance via the downregulation of tight junction (TJ), gap junctional intercellular communication (GJIC), and membrane transporters-organic-aniontransporting polypeptides (OATPs). DEHP shifted the microbial community and microbial capacity to generate bioactive metabolites. This finding suggests that the gut microbiota may contribute to the adverse effects of DEHP on the host by altering metabolites sensed by both intestinal and immune Th cells. DEHP interacts with the expression of intracellular receptors (AhR, PPARs) that regulate cellular metabolism and immunity and downregulates the production of several hormones (GLP-1, GH1, NPY).

the hydrolysis enzyme mix to obtain glucose levels as per the protocol. The glucose background was subtracted from the glycogen readings as per the manufacturer's suggestion to obtain an accurate estimate of total glycogen.

Histological Analysis. Three males and four females from the control group and five males and five females from the DEHP exposed groups at 2 months were collected for histopathology. The GI and liver tissues used for histopathology were preserved in Davidson's fixative, embedded in paraffin, sectioned at $4 \mu \mathrm{M}$, and stained with hematoxylin and eosin. Tissue pathology was assessed by two Veterinary Board-Certified Pathologists (Drs. Ginn and Craft). Scoring of tissue was based upon the severity of changes compared to control sections. Gastrointestinal tissue was evaluated for evidence of disruption of villus morphology, necrosis of the epithelial border, and inflammation. For inflammation, the number of eosinophils at the base of the epithelial cells in the connective tissue stroma was counted in three fields per specimen at $60 \times$ magnification, and the presence of migrating lymphocytes was compared between control and experimental groups. Liver tissue was evaluated for disruption of liver architecture, presence and degree of hepatocellular lipid accumulation, inflammation, bile duct hyperplasia, fibrosis, hepatocyte hypertrophy, and areas of necrosis. The histological scoring system was adapted from Zodrow et al. ${ }^{36}$

\section{RESULTS AND DISCUSSION}

Epidemiological studies link DEHP with numerous adverse health outcomes. ${ }^{9}$ However, there are no data investigating the direct role of the microbiome in DEHP-induced toxicity. It is noteworthy that DEHP has been reported to affect the adaptive immune system as a promoter of Th1/Th2 immune responses. ${ }^{37,38}$ Earlier studies have also reported that DEHP may contribute to allergic reactions and act as an adjuvant in promoting Th2 allergic responses. ${ }^{39}$ To better understand the effects of DEHP on these biological processes and the intestinal immune system, and to reveal interactions between the host gastrointestinal tissue and the microbiome during exposure, we leveraged computational approaches and predictive functional models using multiomics data collected in male and female zebrafish exposed to dietary DEHP (Figure 1).

To describe the adverse outcome of dietary exposure to DEHP, we exposed zebrafish to $3 \mathrm{mg}$ of DEHP $/ \mathrm{kg}$ of feed. The DEHP content in the feed was confirmed with LC MS/ MS $(2.89 \pm 0.06 \mathrm{mg} / \mathrm{kg})$. However, while DEHP is associated with increased adipogenesis, ${ }^{13}$ two month exposure did not alter body weight (Figure S2) nor did it induce pathology in the gastrointestinal system or liver (Figures S3 and S4, Table S1). This has also been reported in a second exposure study with DEHP in zebrafish, in that $3 \mathrm{ppm}$ DEHP did not exacerbate gastrointestinal morphology nor affect BMI during a high-feeding regime. ${ }^{23}$ No change in glucose nor glycogen 
stores in the liver supports the observation that DEHP at these realistic exposures may not affect BMI.

Despite a lack of change in higher level responses, there were many at the molecular level. Gene set enrichment analysis (GSEA) identified classes of genes that were dysregulated by DEHP, several of which are associated with Th cells in both males and females (Table.1). T-cell populations consist of

Table 1. Transcriptomic Analysis of the Intestine of Zebrafish Males and Females Exposed to DEHP ${ }^{a}$

\begin{tabular}{|c|c|c|c|c|}
\hline \multicolumn{5}{|c|}{ gene set enrichment analysis (GSEA) } \\
\hline group & pathway/process name & $\begin{array}{l}\text { normalized } \\
\text { enrichment } \\
\text { score }\end{array}$ & $\begin{array}{l}\text { median } \\
\text { change }\end{array}$ & $\begin{array}{c}p \\
\text { value }\end{array}$ \\
\hline males & $\mathrm{T}$ cell activation & 1.23 & 1.09 & 0.00 \\
\hline males & T-cell receptor signaling & 1.31 & 1.09 & 0.01 \\
\hline males & $\begin{array}{l}\text { Th1 cell activation in Crohn's } \\
\text { disease }\end{array}$ & 1.64 & 1.27 & 0.02 \\
\hline males & $\begin{array}{l}\text { Th17 cell activation in } \\
\text { Crohn's disease }\end{array}$ & 1.75 & 1.25 & 0.01 \\
\hline males & Th17 cell differentiation & 1.37 & 1.20 & 0.04 \\
\hline males & $\begin{array}{l}\text { Th17 cell function in } \\
\text { systemic lupus } \\
\text { erythematosus }\end{array}$ & 1.58 & 1.25 & 0.01 \\
\hline males & $\begin{array}{l}\text { Th0 cell aberrant activation in } \\
\text { systemic lupus } \\
\text { erythematosus }\end{array}$ & 1.46 & 1.15 & 0.01 \\
\hline males & central $\mathrm{T}$-cell tolerance & 1.39 & 1.14 & 0.04 \\
\hline males & $\begin{array}{l}\text { peripheral T-cell tolerance } \\
\text { overview }\end{array}$ & 1.36 & 1.19 & 0.05 \\
\hline males & $\begin{array}{l}\text { T-cell positive selection and } \\
\text { neglect-induced death }\end{array}$ & 1.65 & 1.23 & 0.01 \\
\hline females & $\begin{array}{l}\text { T-cell receptor } \rightarrow \text { AP-1 } \\
\text { signaling }\end{array}$ & 1.52 & 1.18 & 0.03 \\
\hline females & T-cell receptor signaling & -1.28 & -1.05 & 0.03 \\
\hline females & $\begin{array}{l}\text { interleukin-22 induced } \\
\text { keratinocyte proliferation in } \\
\text { psoriasis }\end{array}$ & 1.65 & 1.18 & 0.01 \\
\hline females & IL10R $\rightarrow$ STAT signaling & 1.71 & 1.21 & 0.01 \\
\hline females & IL12R $\rightarrow$ STAT signaling & 1.82 & 1.21 & 0.01 \\
\hline females & IL13R $\rightarrow$ STAT signaling & 1.82 & 1.22 & 0.01 \\
\hline females & IL22R $\rightarrow$ STAT3 signaling & 1.65 & 1.84 & 0.02 \\
\hline females & IL31R $\rightarrow$ STAT signaling & 1.63 & 1.14 & 0.03 \\
\hline \multicolumn{5}{|c|}{ subnetwork analysis (cell processes) } \\
\hline group & pathway/process name & \multicolumn{2}{|c|}{ median change } & $p$ value \\
\hline males & T-helper 1 cell activation & \multicolumn{2}{|c|}{1.27} & 0.03 \\
\hline males & T-helper 17 cell differentiation & \multicolumn{2}{|c|}{1.18} & 0.04 \\
\hline males & T-cell tolerance & \multicolumn{2}{|c|}{1.13} & 0.05 \\
\hline males & resting $\mathrm{T}$-cell proliferation & \multicolumn{2}{|c|}{1.27} & 0.04 \\
\hline males & adaptive immune response & \multicolumn{2}{|c|}{1.05} & 0.03 \\
\hline females & T-helper 2 cell differentiation & \multicolumn{2}{|c|}{1.09} & 0.04 \\
\hline
\end{tabular}

${ }^{a_{T}}$ The table represents a summary of the T cell specific pathways and processes identified by gene set enrichment analysis (GSEA) and cell processes identified by a sub-network analysis. The complete list of identified effected processes and pathways is in the Supporting Information (Table S2 females and Table S3 males).

helper T cell types Th1 and Th2, and their dysregulation is a marker of inflammatory disease states (Crohn's disease is associated with a Th1 cytokine profile, ulcerative colitis with dysregulation of Th2). ${ }^{40}$ Another type of T cell, Th17, has an important role in intestinal inflammation, particularly in Crohn's disease. ${ }^{41}$ Our computational analysis revealed that DEHP upregulates the spectrum of the Th cell cytokines specific for Th1 (Il-12, Il-10, Il-22, INF $\gamma$ ), Th2 (Il-13, Il-31), and Th17 (Il-22; Table S2). Similarly, the intestinal response in the male group suggested there was activation and differentiation of Th cells ( $\mathrm{T}$ cell activation, Th17 cell differentiation), similar to activation observed in Crohn's disease (Table S3). The activation of intestinal populations of Th cells is supported by differential gene expression analysis (DGEA) that revealed that the highly affected gene is nfil3 (Nuclear Factor, Interleukin 3), which was upregulated by DEHP in both males (fold change 15.3) and females (fold change 10.1; Table S4). NFIL3 regulates Il-3 transcription, a cytokine that is restricted to activated $\mathrm{T}$ cells, natural killer (NK) cells, and mast cell lines. The upregulation of $n f i l 3$ by DEHP was verified by qPCR which showed the increased level of $n f i l 3$ transcript (Figure S1). DEHP induced T cell (Th1, Th2, and Th17) activation and differentiation was also observed in our previous study that evaluated the effect of DEHP on metabolism and contribution to obesity. ${ }^{23}$

Th17 plays a key role in the neutralization of pathogens, partly by the maintenance of the integrity of the intestinal epithelia via Il-17 and Il-22 production. The observed cytokine profile, specifically Il-22, suggests that there may be maintenance of epithelial barriers and promotion of the repair of damaged epithelia. ${ }^{42}$ Additionally, mucosal epithelia express receptors for Th17 cytokines, promoting epithelial barrier function by tight junction formation and antimicrobial peptide and mucus production. ${ }^{42}$ Involvement of the pro-inflammatory INF $\gamma$ and Il-31 cytokines is a signature of activated Th cells regulating intestinal barrier function, specifically tight junctions. ${ }^{43}$ Epithelial and endothelial barrier function is mostly maintained by intercellular tight junctions (TJs), that is, multiprotein complexes that seal the space between adjacent cells. Studies have highlighted the regulation of the tight junction barrier by cytokines. ${ }^{44}$ Upregulated IFN- $\gamma$ and Il-22, in addition to other cytokines, e.g., tumor necrosis factor- $\alpha$ $(\mathrm{TNF} \alpha)$, are known for their role in the regulation of tight junction integrity. ${ }^{43,45,46}$ Cytokine mediated perturbation of the function of $\mathrm{TJ}$ may result in enhanced paracellular permeability and increased exposure of tissues to luminal antigens in organ systems. GSEA revealed that DEHP downregulates gene networks associated with the organization and function of cell-cell junctions primarily involved in adhesion, specifically tight junctions (TJs) and adherent junctions (AJ), and gut homeostasis represented by gap junctions (GJs; Tables S2 and S3). GJ creates the pores coupling adjacent cells to mediate intercellular activities of GJ called gap junction intercellular communication (GJIC). Interestingly, the expression of GCs in females (i.e., GC assembly and regulation) is downregulated (Table S4). This finding is supported by significant downregulation of connexin 32.3 (cx32.3), the transcript of the key GC structure peptide (Table S5). Inhibition of GJIC may impact the exchange of small molecules between adjacent cells that is critical for maintaining tissue homeostasis and integrity. We assume that the perturbation of GJIC and TJs is caused by a stimulation of the immune system. ${ }^{44,47}$ However, the effect of phthalates on cell-cell communication and $\mathrm{TJ}$ and related mode of action is largely unexplored. There are a few studies demonstrating that similar types of phthalate, for example, di- $n$-butyl phthalate, induce structural and function changes of TJs and GJs in the testes, both in vivo and in vitro. ${ }^{48,49}$ However, the mode of action underlying the disruption is not yet firmly established.

Aberrant immune signals derived from DEHP exposure, in addition to the associated changes in intestinal barrier function 


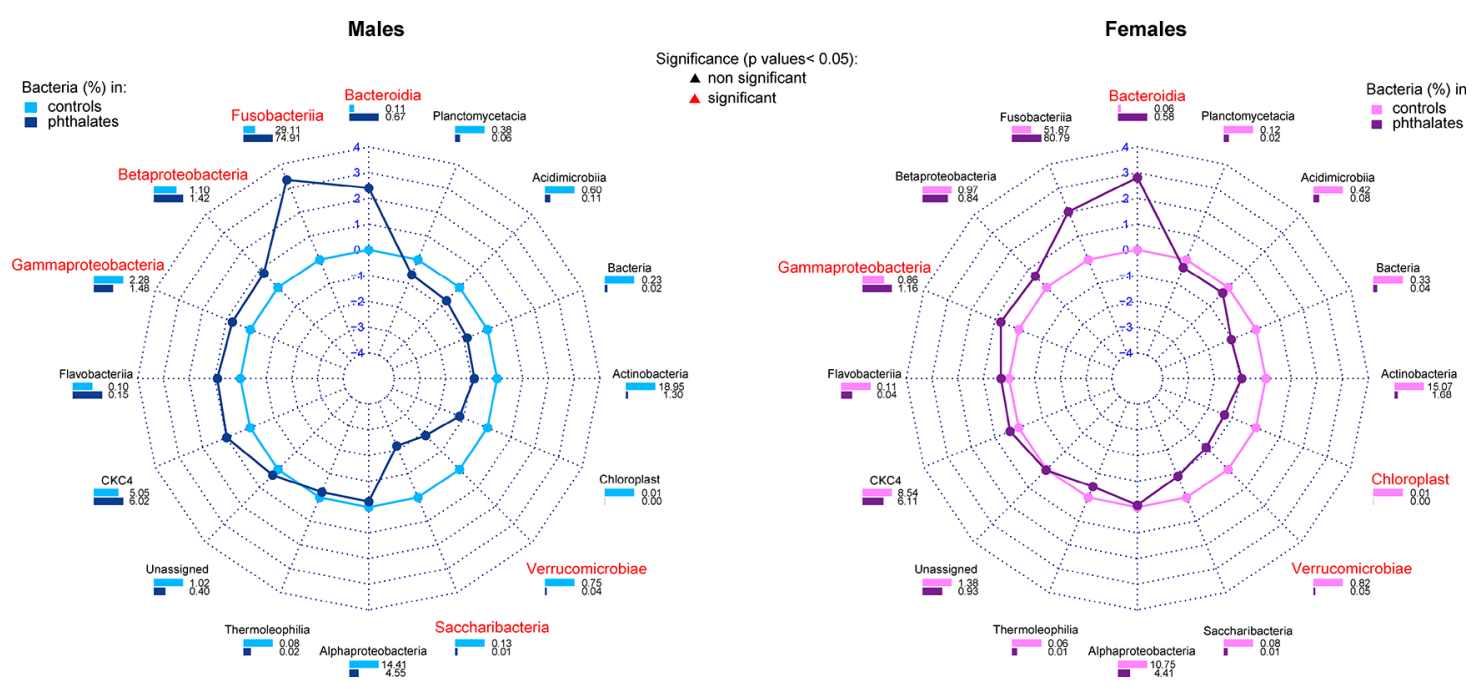

Figure 2. Comparison of the effect of phthalate DEHP on microbial classes between control and exposed group in male and female groups. Radar charts represent the shift in the specific microbial class expressed as the difference between median abundance of control and exposed groups. The bars under each microbial class represent the precentral abundance (\%) of each class within the control or exposed group. Statistically significant effects on the abundance of the microbial class is marked in red.

and integrity, may contribute to dysfunction as revealed via the subnetwork analysis. The intestinal transcriptomic profile suggests several common effects for male and female groups on tissue maintenance, gut development, GI digestion and secretion, and duodenum motility, among others. There also appears to be sex specific effects for males in terms of intestinal transportation and absorption capacity (e.g., effect on lipid transport and absorption and fatty acid import) and for females (e.g., intestinal secretion, wall integrity and GI transit; Tables S2, S3). Moreover, we identify the impact on overall performance of the gut, as several membrane transport proteins, organic-anion-transporting polypeptides (OATPs), encoded by $s l c$ genes were negatively affected in the males (Table S3). OATPs are evolutionary ancient proteins able to transport ions, hormones, nutrients, and xenobiotics and are a key player for oral drug absorption. ${ }^{50}$ Alterations in the expression and function of OATP drug transporters have been reported to occur in several disease states including cancer and inflammatory diseases. ${ }^{51}$ Several human studies with hepatocytes demonstrate a role for the immune system in the regulation of OATP. Pro-inflammatory TNF- $\alpha$, IL-6, oncostatin, IL- $1 \beta$, and IFN- $\gamma$ downregulate or repress a wide spectrum of OATPs partially through transcription factors HNF $1 \alpha$ (hepatocyte nuclear factor-1 $\alpha$ ) and HNF $4 \alpha$ (hepatocyte nuclear factor- $4 \alpha) .^{51}$

In addition to GSEA, SNEA confirmed the effect on immune cells emphasizing the involvement of T cells; as many of the identified targets are $\mathrm{T}$ cell cytokines or $\mathrm{T}$ cell receptors of chemokines or are those that belong to transduction pathways that lead to cytokine production. We also identified downregulation of several hormone networks and/or their receptor signaling pathways such as growth hormone 1 (GH1), glucagon-like peptide-1 (GLP-1), and neuropeptide Y (NPY; Tables S2, S3) at the transcript level. NPY is one of the hormones that can modify immune activity. NPY is an agonist of $\mathrm{Y}$ class receptor that is expressed in specific immune cells such as macrophages, dendritic cells, and $\mathrm{T}$ and $\mathrm{B}$ cells. ${ }^{52}$ In addition, immune cells including $\mathrm{B}$ and $\mathrm{T}$ lymphocytes release NPY as an autocrine and paracrine immune mediator. ${ }^{53} \mathrm{NPY}$ negatively regulates $\mathrm{T}$ cell function, activates $\mathrm{Th}$ cell differentiation, and can modulate immune cell trafficking, production of reactive oxygen species, cytokine secretion, and phagocytosis. ${ }^{52,54}$ There is evidence that the NPY system has an impact on the composition and function of the gut microbiota. ${ }^{52}$ NPY has been found to exhibit a direct antimicrobial effect against various gut bacteria including Escherichia, Enterococcus, and Lactobacillus. ${ }^{55}$ On the contrary, the microbiome influences diverse aspects of intestinal physiology and disease, in part by controlling tissue-specific transcription of host genes. One of the identified DEHP affected transcription factors in the intestine were HNF $4 \alpha$ and $\operatorname{HNF} 1 \alpha$, each of which play an important role in human metabolic and inflammatory bowel diseases. ${ }^{56}$ Recently, genetic analysis revealed that zebrafish $h n f 4 a$ activates nearly half of the genes that are suppressed by microbiota, suggesting microbiota negatively regulate $\mathrm{HNF} 4 \alpha .{ }^{56}$

Activation of an intestinal immune system and the activation of microbiome responsive elements appears to underlie DEHP exposure. We thus conducted a metagenomic analysis of the intestinal microbiota, as DEHP was hypothesized to shift the microbiome composition and decrease diversity. Specifically, we observed an increase in phyla Fusobacteria, Bacteroidetes, and Verrucomicrobia in both males and females (Figures S5 and S6). On a class level, DEHP exposure increased Bacteroidia and Gammaproteobacteria and decreased Verrucomicrobiae in both sexes. Further, in males we found an increase in classes Fusobacteriia and Betaproteobacteria and a decrease in Saccharibacteria (Figure 2). The shift of the microbiome does not necessarily reflect the change in microbial functional capabilities. $^{30}$ A computational approach that predicts the functional composition of the metagenome revealed metabolic and biosynthetic pathways involved in the production of bioactive metabolites. In contrast to males, the predicted microbial functional shift in females involved metabolismrelated categories (e.g., decreased oxidative phosphorylation and benzoate degradation; Figure S7). In males, there was a predicted increased capacity for glycerophospholipid metabolism, proposed to be associated with choline and lower tryptophan metabolism (Figure S8). We next used predicted community enzyme function profiling to model microbial 


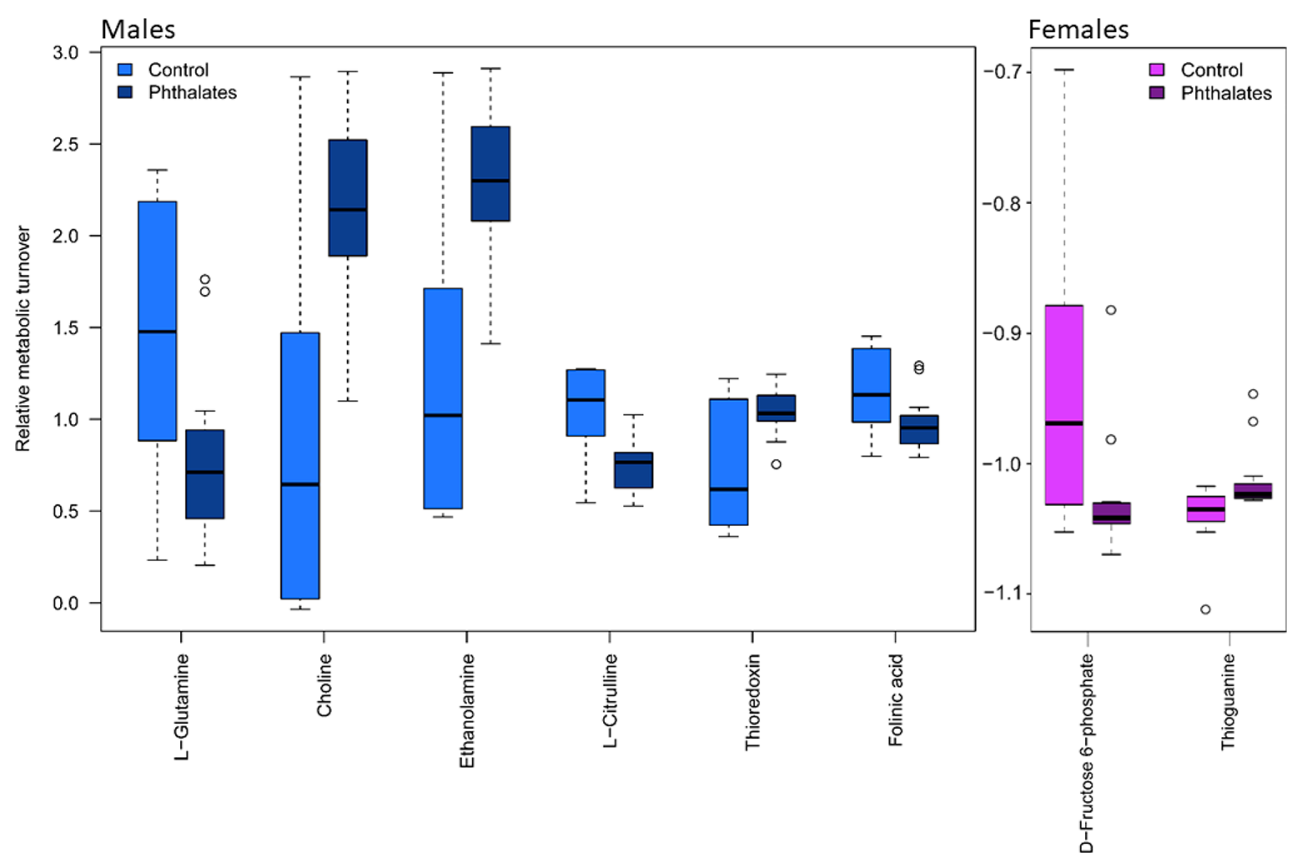

Figure 3. Significant changes in predicted relative metabolic turnover (PRMT) of microbial metabolites in control and DEHP exposed (phthalates) male and female groups.

metabolites using Predicted Relative Metabolic Turnover (PRMT; ${ }^{57,58}$ Figure 3 ). Our model showed several microbial metabolites that may, in addition to a direct effect of DEHP, contribute to immune and intercellular communication. Our predictions revealed decreased L-glutamine in males and Dfructose 6-phosphate in females; these metabolites are beneficial to diverse immune functions. Moreover, these metabolites require precise control of cellular metabolic pathways and impact immune cells and enterocytes. For example, lower L-glutamine may impact L-citrulline levels, a biomarker of short bowel syndrome (SBS) ${ }^{59}$ Citrulline is also lower in adenovirus enteritis and all infectious intestinal diseases with high cytopathic effects. ${ }^{60}$

Further, we found a predicted increase in the turnover of metabolites with immunomodulatory potency, specifically choline in the males and thioguanine (TG) in females (Figure 3). Choline is actively metabolized to methylamines (e.g., trimethylamine-N-oxide; TMAO) which have been shown to be involved in many diseases such as obesity, diabetes, and cancers including autoimmune deseases. ${ }^{61}$ TMAO upregulates the inflammatory pathways leading to increased production of pro-inflammatory cytokines. ${ }^{62} \mathrm{TG}$ is a member of thiopurines, an active ingredient of IBD drugs, that induce immunosuppression, one of which reduces proliferation of cancerous cells. ${ }^{63}$ In addition to positive effects on IBD, these chemicals induce oxidative stress and apoptosis specifically in $\mathrm{Th}$ cells. $^{63-65}$ On the basis of the response in the zebrafish intestine, we posit that tryptophan metabolites may also play a role in immune related effects. However, GSEA identified the increased tryptophan metabolism in the intestine; we found a predicted decrease of tryptophan metabolic turnover in microbial communities. Tryptophan metabolites are converted from tryptophan in the lumen of the gut and regulate arylhydrocarbon receptor (AhR) signaling in a broad spectrum of cells. The AhR is a ligand-inducible transcription factor/ receptor that is highly expressed by epithelial cells and immune cells, both the Il-17/Il-22 producing and nonproducing Th cells. $^{66,67}$ Tryptophan levels thus regulate $\mathrm{T}$ cells (i.e., regulators of differentiation of $\mathrm{T}$ lymphocytes into proinflammatory Th 17 cells) and promote AhR-dependent production of Il-22, a key cytokine involved in maintenance of epithelial barriers and repair processes. ${ }^{68}$ Further, there are additional metabolites that link the effect on Th cells and the dysregulated microbiome, thioredoxin, that is part of thioredoxin system ( $\operatorname{Trx}$ ) that is suggested to be the critical pathway controlling $\mathrm{T}$-cell activation and expansion. ${ }^{69}$ Upregulated Trx has been reported in several types of cancers and deregulated $\mathrm{T}$ cells (e.g., aggressive T-cell lymphoblastic leukemia). ${ }^{69}$

The observed effects, i.e., dysregulated genes and biological processes, are proposed to be early events that may lead to DEHP-induced abnormalities. We evaluated several apical end points to connect the early events to specific adverse outcomes. In spite of a dramatic shift in several biomarkers of the microbiome-gut axis, there were no histological differences between control and DEHP exposed animals in the liver or gut tissue (Figures S3 and S4, Table S1). To link the observed effect with DEHP associated lipid disbalance and increase in energy storage, we measured weight gain (Figure S2) and glucose and glycogen (Figure S9) levels in livers. We did not detect any significant changes among control and DEHPexposed animals, and there was high individual variability in glucose and glycogen levels.

In conclusion, we leverage a multiomics approach (e.g., transcriptomic analysis with metagenomics) to systematically obtain a deeper understanding into the processes that may lead to adverse outcomes reported in other studies with plasticizers. We report that DEHP modulates several biological processes that affect complex intestinal performance. We assume that these effects are due in part to a direct effect of DEHP on the intestine but are also mediated via dysregulated adaptive immunity, specifically $\mathrm{Th}$ cells. The latest advances in bioinformatics allowed us to predict the effects of DEHP on microbiome-gut mutual signaling and to confirm the role of 
microbial bioactive metabolites on the dysregulation of the host immune system. The identified immunity-microbiome network altered by DEHP is expected to lead to a deeper understanding of plasticizers and adverse health outcomes identified in human cohort studies.

\section{ASSOCIATED CONTENT}

\section{SI Supporting Information}

The Supporting Information is available free of charge at https://pubs.acs.org/doi/10.1021/acs.est.0c00628.

Microarrays and PCR results of selected genes, body weights, histopathology, microbial phyla (radar chart, PCA plot), microbial functional shift, glycogen levels in liver, transcriptomic analysis (GSEA, SNEA), GSEA focused on Gap junction intracellular communication and Gap junction intracellular assembly, differentially expressed genes (PDF)

The results of the gene set enrichment analysis (GSEA) and sub-network analysis (SNEA) identifying impact on cell processes and other targets in the female intestine after 2 months exposure to DEHP (XLSX)

The results of the gene set enrichment analysis (GSEA) and sub-network analysis (SNEA) identifying impact on cell processes and other targets in the male intestine after 2 months exposure to DEHP (XLSX)

DEHP induced disruptive effect on a gene network associated with intestinal gap junction intercellular communication (GJIC assembly and regulation) in female zebrafish identified using gene set enrichment analysis (XLSX)

Differentially expressed genes in male and female zebrafish exposed to DEHP for two months (XLSX)

\section{AUTHOR INFORMATION}

\section{Corresponding Authors}

Joseph H. Bisesi, Jr. - Department of Environmental and Global Health and Center for Environmental and Human Toxicology, College of Public Health and Health Professions, University of Florida, Gainesville, Florida, United States; Email: jbisesi@phhp.ufl.edu

Christopher J. Martyniuk - Department of Physiological Sciences and Center for Environmental and Human Toxicology, UF Genetics Institute, College of Veterinary Medicine, University of Florida, Gainesville, Florida 32611, United States; Email: cmartyn@ufl.edu

\section{Authors}

Ondrej Adamovsky - Research Centre for Toxic Compounds in the Environment (RECETOX), Masaryk University, Brno, Czech Republic; Department of Physiological Sciences and Center for Environmental and Human Toxicology, UF Genetics Institute, College of Veterinary Medicine, University of Florida,

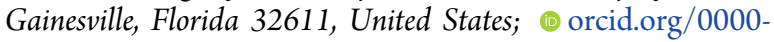
0003-3084-0388

Amanda N. Buerger - Department of Environmental and Global Health and Center for Environmental and Human Toxicology, College of Public Health and Health Professions, University of Florida, Gainesville, Florida, United States

Hana Vespalcova - Research Centre for Toxic Compounds in the Environment (RECETOX), Masaryk University, Brno, Czech Republic
Shahadur R. Sohag - Department of Physiological Sciences and Center for Environmental and Human Toxicology, UF Genetics Institute, College of Veterinary Medicine, University of Florida, Gainesville, Florida 32611, United States

Amy T. Hanlon - Department of Physiological Sciences and Center for Environmental and Human Toxicology, UF Genetics Institute, College of Veterinary Medicine, University of Florida, Gainesville, Florida 32611, United States

Pamela E. Ginn - Department of Comparative, Diagnostic and Population Medicine, College of Veterinary Medicine, University of Florida, Gainesville, Florida, United States

Serena L. Craft - Department of Comparative, Diagnostic and Population Medicine, College of Veterinary Medicine, University of Florida, Gainesville, Florida, United States

Stanislav Smatana - Research Centre for Toxic Compounds in the Environment (RECETOX), Masaryk University, Brno, Czech Republic; Brno University of Technology, Faculty of Information Technology, IT4Innovations Centre of Excellence, 61266 Brno, Czech Republic

Eva Budinska - Research Centre for Toxic Compounds in the Environment (RECETOX), Masaryk University, Brno, Czech Republic

Maria Persico - Research Centre for Toxic Compounds in the Environment (RECETOX), Masaryk University, Brno, Czech Republic

Complete contact information is available at:

https://pubs.acs.org/10.1021/acs.est.0c00628

\section{Notes}

The authors declare no competing financial interest.

\section{ACKNOWLEDGMENTS}

This project received funding from the European Union's Horizon 2020 research and innovation programme under the Marie Sklodowska-Curie grant agreement no. 707241 (OA). The project was also supported by RECETOX research infrastructure (the Czech Ministry of Education, Youth and Sports: LM2018121) and by Teaming project: CETOCOEN EXCELLENCE Teaming 2 project supported by Horizon 2020 (857560) and the Czech ministry of Education, Youth and Sports (02.1.01/0.0/0.0/18_046/0015975). Bioinformatics were supported by Brno Unniversity of Technology, grant FIT-S-17-3994. Computational resources were supplied by the Ministry of Education, Youth and Sports of the Czech Republic under the Projects CESNET (Project No. LM2015042) and CERIT-Scientific Cloud (Project No. LM2015085) provided within the program Projects of Large Research, Development and Innovations Infrastructures. Transcriptomics was supported by University of Florida Start Up Fund (C.J.M.).

\section{REFERENCES}

(1) ECHA Europe. Report: Submission of Information on DINP; Helsinky, Finland, 2014.

(2) Deutschle, T.; Reiter, R.; Butte, W.; Heinzow, B.; Keck, T.; Riechelmann, H. A Controlled Challenge Study on Di(2-Ethylhexyl) Phthalate (DEHP) in House Dust and the Immune Response in Human Nasal Mucosa of Allergic Subjects. Environ. Health Perspect. 2008, 116 (11), 1487-1493.

(3) Schwartz, H. E.; Anzion, S. C. J. M.; Van Vliet, S. H. P. M.; Copius Peerebooms, J. W.; Brinkman, U. A. T. Analysis of Phthalate Esters in Sediments from Dutch Rivers by Means of High 
Performance Liquid Chromatography. Int. J. Environ. Anal. Chem. 1979, 6 (2), 133-144.

(4) Tranfo, G.; Caporossi, L.; Pigini, D.; Capanna, S.; Papaleo, B.; Paci, E. Temporal Trends of Urinary Phthalate Concentrations in Two Populations: Effects of REACH Authorization after Five Years. Int. J. Environ. Res. Public Health 2018, 15 (9), 1950.

(5) Koch, H. M.; Lorber, M.; Christensen, K. L. Y.; Pälmke, C.; Koslitz, S.; Brüning, T. Identifying Sources of Phthalate Exposure with Human Biomonitoring: Results of a 48h Fasting Study with Urine Collection and Personal Activity Patterns. Int. J. Hyg. Environ. Health 2013, 216 (6), 672-681.

(6) Serrano, S. E.; Braun, J.; Trasande, L.; Dills, R.; Sathyanarayana, S. Phthalates and Diet: A Review of the Food Monitoring and Epidemiology Data. Environ. Health 2014, 13 (1), 1-14.

(7) Cao, X.-L. Phthalate Esters in Foods: Sources, Occurrence, and Analytical Methods. Compr. Rev. Food Sci. Food Saf. 2010, 9 (1), 2143.

(8) Kelley, K. E.; Hernández-Díaz, S.; Chaplin, E. L.; Hauser, R.; Mitchell, A. A. Identification of Phthalates in Medications and Dietary Supplement Formulations in the United States and Canada. Environ. Health Perspect. 2012, 120 (3), 379-384.

(9) Benjamin, S.; Masai, E.; Kamimura, N.; Takahashi, K.; Anderson, R. C.; Faisal, P. A. Phthalates Impact Human Health: Epidemiological Evidences and Plausible Mechanism of Action. J. Hazard. Mater. 2017, 340, 360-383.

(10) Trasande, L.; Attina, T. M.; Sathyanarayana, S.; Spanier, A. J.; Blustein, J. Race/Ethnicity-Specific Associations of Urinary Phthalates with Childhood Body Mass in a Nationally Representative Sample. Environ. Health Perspect. 2013, 121 (4), 501-506.

(11) Teitelbaum, S. L.; Mervish, N.; Moshier, E. L.; Vangeepuram, N.; Galvez, M. P.; Calafat, A. M.; Silva, M. J.; Brenner, B. L.; Wolff, M. S. Associations between Phthalate Metabolite Urinary Concentrations and Body Size Measures in New York City Children. Environ. Res. 2012, 112, 186-193.

(12) Hatch, E. E.; Nelson, J. W.; Qureshi, M. M.; Weinberg, J.; Moore, L. L.; Singer, M.; Webster, T. F. Association of Urinary Phthalate Metabolite Concentrations with Body Mass Index and Waist Circumference: A Cross-Sectional Study of NHANES Data, 1999-2002. Environ. Health 2008, 7 (1), 27.

(13) Lind, P. M.; Roos, V.; Rönn, M.; Johansson, L.; Ahlström, H.; Kullberg, J.; Lind, L. Serum Concentrations of Phthalate Metabolites Are Related to Abdominal Fat Distribution Two Years Later in Elderly Women. Environ. Health 2012, 11 (1), 21.

(14) Kuo, C. H.; Hsieh, C. C.; Kuo, H. F.; Huang, M. Y.; Yang, S. N.; Chen, L. C.; Huang, S. K.; Hung, C. H. Phthalates Suppress Type i Interferon in Human Plasmacytoid Dendritic Cells via Epigenetic Regulation. Allergy 2013, 68 (7), 870-879.

(15) Shi, W.; Lin, Z.; Liao, C.; Zhang, J.; Liu, W.; Wang, X.; Cai, J.; Zou, Z.; Wang, H.; Norback, D.; Kan, H.; Huang, C.; Zhao, Z. Urinary Phthalate Metabolites in Relation to Childhood Asthmatic and Allergic Symptoms in Shanghai. Environ. Int. 2018, 121, 276286.

(16) Jaakkola, J. J. K.; Knight, T. L. The Role of Exposure to Phthalates from Polyvinyl Chloride Products in the Development of Asthma and Allergies: A Systematic Review and Meta-Analysis. Environ. Health Perspect. 2008, 116 (7), 845-853.

(17) Han, Y.; Wang, X.; Chen, G.; Xu, G.; Liu, X.; Zhu, W.; Hu, P.; Zhang, Y.; Zhu, C.; Miao, J. Di-(2-Ethylhexyl) Phthalate Adjuvantly Induces Imbalanced Humoral Immunity in Ovalbumin-Sensitized BALB/c Mice Ascribing to T Follicular Helper Cells Hyperfunction. Toxicology 2014, 324, 88-97.

(18) Alfardan, A. S.; Nadeem, A.; Ahmad, S. F.; Al-Harbi, N. O.; AlHarbi, M. M.; AlSharari, S. D. Plasticizer, Di(2-Ethylhexyl)Phthalate (DEHP) Enhances Cockroach Allergen Extract-Driven Airway Inflammation by Enhancing Pulmonary Th2 as Well as Th17 Immune Responses in Mice. Environ. Res. 2018, 164, 327-339.

(19) Boulangé, C. L.; Neves, A. L.; Chilloux, J.; Nicholson, J. K.; Dumas, M.-E. Impact of the Gut Microbiota on Inflammation, Obesity, and Metabolic Disease. Genome Med. 2016, 8 (1), 42.
(20) Lu, K.; Mahbub, R.; Fox, J. G. Xenobiotics: Interaction with the Intestinal Microflora. ILAR J. 2015, 56 (2), 218-227.

(21) Hu, J.; Raikhel, V.; Gopalakrishnan, K.; Fernandez-Hernandez, H.; Lambertini, L.; Manservisi, F.; Falcioni, L.; Bua, L.; Belpoggi, F.; Teitelbaum, S. L.; Chen, J. Effect of Postnatal Low-Dose Exposure to Environmental Chemicals on the Gut Microbiome in a Rodent Model. Microbiome 2016, 4 (1), 26.

(22) U.S. Department of Health and Human Services. Toxicological Profile for Di(2-Ethylhexyl)Phthalate (DEHP); Atlanta, GA, 2019.

(23) Buerger, A. N.; Schmidt, J.; Chase, A.; Paixao, C.; Patel, T. N.; Brumback, B. A.; Kane, A. S.; Martyniuk, C. J.; Bisesi, J. H. Examining the Responses of the Zebrafish (Danio Rerio) Gastrointestinal System to the Suspected Obesogen Diethylhexyl Phthalate. Environ. Pollut. 2019, 245, 1086-1094.

(24) Loughery, J. R.; Marentette, J. R.; Frank, R. A.; Hewitt, L. M.; Parrott, J. L.; Martyniuk, C. J. Transcriptome Profiling in Larval Fathead Minnow Exposed to Commercial Naphthenic Acids and Extracts from Fresh and Aged Oil Sands Process-Affected Water. Environ. Sci. Technol. 2019, 53 (17), 10435-10444.

(25) Bolger, A. M.; Lohse, M.; Usadel, B. Trimmomatic: A Flexible Trimmer for Illumina Sequence Data. Bioinformatics 2014, 30 (15), 2114-2120.

(26) Edgar, R. C. Search and Clustering Orders of Magnitude Faster than BLAST. Bioinformatics 2010, 26 (19), 2460-2461.

(27) Caporaso, J G.; Kuczynski, J.; Stombaugh, J.; Bittinger, K.; Bushman, F. D; Costello, E. K; Fierer, N.; Pena, A. G.; Goodrich, J. K; Gordon, J. I; Huttley, G. A; Kelley, S. T; Knights, D.; Koenig, J. E; Ley, R. E; Lozupone, C. A; McDonald, D.; Muegge, B. D; Pirrung, M.; Reeder, J.; Sevinsky, J. R; Turnbaugh, P. J; Walters, W. A; Widmann, J.; Yatsunenko, T.; Zaneveld, J.; Knight, R. QIIME Allows Analysis of High-Throughput Community Sequencing Data. Nat. Methods 2010, 7, 335-336.

(28) Pruesse, E.; Quast, C.; Knittel, K.; Fuchs, B. M.; Ludwig, W.; Peplies, J.; Glockner, F. O. SILVA: A Comprehensive Online Resource for Quality Checked and Aligned Ribosomal RNA Sequence Data Compatible with ARB. Nucleic Acids Res. 2007, 35 (21), 71887196.

(29) Kanehisa, M. KEGG: Kyoto Encyclopedia of Genes and Genomes. Nucleic Acids Res. 2000, 28 (1), 27-30.

(30) Langille, M. G. I.; Zaneveld, J.; Caporaso, J. G.; McDonald, D.; Knights, D.; Reyes, J. A.; Clemente, J. C.; Burkepile, D. E.; Vega Thurber, R. L.; Knight, R.; Beiko, R. G.; Huttenhower, C. Predictive Functional Profiling of Microbial Communities Using 16S RRNA Marker Gene Sequences. Nat. Biotechnol. 2013, 31 (9), 814-821.

(31) Manor, O.; Borenstein, E. MUSiCC: A Marker Genes Based Framework for Metagenomic Normalization and Accurate Profiling of Gene Abundances in the Microbiome. Genome Biol. 2015, 16 (1), 53.

(32) Larsen, P. E.; Collart, F. R.; Field, D.; Meyer, F.; Keegan, K. P.; Henry, C. S.; McGrath, J.; Quinn, J.; Gilbert, J. A. Predicted Relative Metabolomic Turnover (PRMT): Determining Metabolic Turnover from a Coastal Marine Metagenomic Dataset. Microb. Inf. Exp. 2011, 1 (1), 4.

(33) Noecker, C.; Eng, A.; Srinivasan, S.; Theriot, C. M.; Young, V. B.; Jansson, J. K.; Fredricks, D. N.; Borenstein, E. Metabolic ModelBased Integration of Microbiome Taxonomic and Metabolomic Profiles Elucidates Mechanistic Links between Ecological and Metabolic Variation. mSystems 2016, 1 (1), 1-17.

(34) Aitchison, J. The Statistical Analysis of Compositional Data. In Journal of the Royal Statistical Society. Series B (Methodological); Royal Statistical Society, 1982; Vol. 44, pp 139-177, DOI: 10.2307/ 2982045.

(35) R Development Core Team. R: A Language and Environment for Statistical Computing; 2018, DOI: 10.1007/978-3-540-74686-7.

(36) Zodrow, J. M.; Stegeman, J. J.; Tanguay, R. L. Histological Analysis of Acute Toxicity of 2,3,7,8-Tetrachlorodibenzo-p-Dioxin (TCDD) in Zebrafish. Aquat. Toxicol. 2004, 66 (1), 25-38.

(37) Han, Y.; Wang, X.; Chen, G.; Xu, G.; Liu, X.; Zhu, W.; Hu, P.; Zhang, Y.; Zhu, C.; Miao, J. Di-(2-Ethylhexyl) Phthalate Adjuvantly Induces Imbalanced Humoral Immunity in Ovalbumin-Sensitized 
BALB/c Mice Ascribing to T Follicular Helper Cells Hyperfunction. Toxicology 2014, 324, 88-97.

(38) Larsen, S. T.; Hansen, J. S.; Hansen, E. W.; Clausen, P. A.; Nielsen, G. D. Airway Inflammation and Adjuvant Effect after Repeated Airborne Exposures to Di-(2-Ethylhexyl)Phthalate and Ovalbumin in BALB/c Mice. Toxicology 2007, 235 (1-2), 119-129.

(39) Alfardan, A. S.; Nadeem, A.; Ahmad, S. F.; Al-Harbi, N. O.; AlHarbi, M. M.; AlSharari, S. D. Plasticizer, Di(2-Ethylhexyl)Phthalate (DEHP) Enhances Cockroach Allergen Extract-Driven Airway Inflammation by Enhancing Pulmonary Th2 as Well as Th17 Immune Responses in Mice. Environ. Res. 2018, 164, 327-339.

(40) Vijay-Kumar, M.; Chassaing, B.; Kumar, M.; Baker, M.; Singh, V. Mammalian Gut Immunity. Biomed. J. 2014, 37 (5), 246.

(41) Brand, S. Crohn's Disease: Th1, Th17 or Both? The Change of a Paradigm: New Immunological and Genetic Insights Implicate Th17 Cells in the Pathogenesis of Crohn's Disease. Gut 2009, 58 (8), $1152-1167$.

(42) Abbas, A. K.; Lichtman, A. H.; Pillai, S. Basic Immunology (Book), 6th ed.; Elsevier, 2019.

(43) Tilg, H.; Zmora, N.; Adolph, T. E.; Elinav, E. The Intestinal Microbiota Fuelling Metabolic Inflammation. Nat. Rev. Immunol. 2020, 20 (1), 40-54.

(44) Capaldo, C. T.; Nusrat, A. Cytokine Regulation of Tight Junctions. Biochim. Biophys. Acta, Biomembr. 2009, 1788 (4), 864871.

(45) Chelakkot, C.; Ghim, J.; Ryu, S. H. Mechanisms Regulating Intestinal Barrier Integrity and Its Pathological Implications. Exp. Mol. Med. 2018, 50 (8), 103.

(46) Wang, Y.; Mumm, J. B.; Herbst, R.; Kolbeck, R.; Wang, Y. IL22 Increases Permeability of Intestinal Epithelial Tight Junctions by Enhancing Claudin-2 Expression. J. Immunol. 2017, 199 (9), 33163325.

(47) Willebrords, J.; Crespo Yanguas, S.; Maes, M.; Decrock, E.; Wang, N.; Leybaert, L.; Kwak, B. R.; Green, C. R.; Cogliati, B.; Vinken, M. Connexins and Their Channels in Inflammation. Crit. Rev. Biochem. Mol. Biol. 2016, 51 (6), 413-439.

(48) Hu, Y.; Wang, R.; Xiang, Z.; Qian, W.; Han, X.; Li, D. Mixture Effects of Nonylphenol and Di-n-Butyl Phthalate (Monobutyl Phthalate) on the Tight Junctions between Sertoli Cells in Male Rats in Vitro and in Vivo. Exp. Toxicol. Pathol. 2014, 66 (9-10), $445-454$.

(49) Sobarzo, C. M.; Lustig, L.; Ponzio, R.; Suescun, M. O.; Denduchis, B. Effects of Di(2-Ethylhexyl) Phthalate on Gap and Tight Junction Protein Expression in the Testis of Prepubertal Rats. Microsc. Res. Tech. 2009, 72 (11), 868-877.

(50) Watanabe, M. Gap Junction in the Teleost Fish Lineage: Duplicated Connexins May Contribute to Skin Pattern Formation and Body Shape Determination. Front. Cell Dev. Biol. 2017, 5, 1-8.

(51) Murray, M.; Zhou, F. Trafficking and Other Regulatory Mechanisms for Organic Anion Transporting Polypeptides and Organic Anion Transporters That Modulate Cellular Drug and Xenobiotic Influx and That Are Dysregulated in Disease. Br. J. Pharmacol. 2017, 174 (13), 1908-1924.

(52) Holzer, P.; Farzi, A. Microbial Endocrinology: The MicrobiotaGut-Brain Axis in Health and Disease; Lyte, M., Cryan, J. F., Eds.; Advances in Experimental Medicine and Biology; Springer: New York, 2014; Vol. 817, DOI: 10.1007/978-1-4939-0897-4.

(53) Farzi, A.; Reichmann, F.; Holzer, P. The Homeostatic Role of Neuropeptide $\mathrm{Y}$ in Immune Function and Its Impact on Mood and Behaviour. Acta Physiol. 2015, 213 (3), 603-627.

(54) Murphy, K. G.; Bloom, S. R. Gut Hormones and the Regulation of Energy Homeostasis. Nature 2006, 444 (7121), 854-859.

(55) El Karim, I. A.; Linden, G. J.; Orr, D. F.; Lundy, F. T. Antimicrobial Activity of Neuropeptides against a Range of MicroOrganisms from Skin, Oral, Respiratory and Gastrointestinal Tract Sites. J. Neuroimmunol. 2008, 200 (1-2), 11-16.

(56) Davison, J. M.; Lickwar, C. R.; Song, L.; Breton, G.; Crawford, G. E.; Rawls, J. F. Microbiota Regulate Intestinal Epithelial Gene
Expression by Suppressing the Transcription Factor Hepatocyte Nuclear Factor 4 Alpha. Genome Res. 2017, 27 (7), 1195-1206.

(57) Larsen, P. E.; Scott, N.; Post, A. F.; Field, D.; Knight, R.; Hamada, Y.; Gilbert, J. A. Satellite Remote Sensing Data Can Be Used to Model Marine Microbial Metabolite Turnover. ISME J. 2015, 9 (1), 166-179.

(58) Larsen, P. E.; Dai, Y. Metabolome of Human Gut Microbiome Is Predictive of Host Dysbiosis. GigaScience 2015, 4 (1), 42.

(59) Crenn, P.; Coudray-Lucas, C.; Thuillier, F.; Cynober, L.; Messing, B. Postabsorptive Plasma Citrulline Concentration Is a Marker of Absorptive Enterocyte Mass and Intestinal Failure in Humans. Gastroenterology 2000, 119 (6), 1496-1505.

(60) Crenn, P.; Messing, B.; Cynober, L. Citrulline as a Biomarker of Intestinal Failure Due to Enterocyte Mass Reduction. Clin. Nutr. 2008, 27 (3), 328-339.

(61) Holmes, E.; Li, J. V.; Athanasiou, T.; Ashrafian, H.; Nicholson, J. K. Understanding the Role of Gut Microbiome-Host Metabolic Signal Disruption in Health and Disease. Trends Microbiol. 2011, 19 (7), 349-359.

(62) Chan, M. M.; Yang, X.; Wang, H.; Saaoud, F.; Sun, Y.; Fong, D. The Microbial Metabolite Trimethylamine N-Oxide Links Vascular Dysfunctions and the Autoimmune Disease Rheumatoid Arthritis. Nutrients 2019, 11 (8), 1821.

(63) Misdaq, M.; Ziegler, S.; von Ahsen, N.; Oellerich, M.; Asif, A. $\mathrm{R}$. Thiopurines Induce Oxidative Stress in T-Lymphocytes: A Proteomic Approach. Mediators Inflammation 2015, 2015, 1-14.

(64) Tiede, I.; Fritz, G.; Strand, S.; Poppe, D.; Dvorsky, R.; Strand, D.; Lehr, H. A.; Wirtz, S.; Becker, C.; Atreya, R.; Mudter, J.; Hildner, K.; Bartsch, B.; Holtmann, M.; Blumberg, R.; Walczak, H.; Iven, H.; Galle, P. R.; Ahmadian, M. R.; Neurath, M. F. CD28-Dependent Rac1 Activation Is the Molecular Target of Azathioprine in Primary Human CD4+ T Lymphocytes. J. Clin. Invest. 2003, 111 (8), 1133-1145.

(65) Kazmers, I. S.; Daddona, P. E.; Dalke, A. P.; Kelley, W. N. Effect of Immunosuppressive Agents on Human $\mathrm{T}$ and $\mathrm{B}$ Lymphoblasts. Biochem. Pharmacol. 1983, 32 (5), 805-810.

(66) Adamovsky, O.; Buerger, A. N.; Wormington, A. M.; Ector, N.; Griffitt, R. J.; Bisesi, J. H.; Martyniuk, C. J. The Gut Microbiome and Aquatic Toxicology: An Emerging Concept for Environmental Health. Environ. Toxicol. Chem. 2018, 37 (11), 2758-2775.

(67) Esser, C.; Rannug, A. The Aryl Hydrocarbon Receptor in Barrier Organ Physiology, Immunology, and Toxicology. Pharmacol. Rev. 2015, 67 (2), 259-279.

(68) Lee, J. S.; Cella, M.; McDonald, K. G.; Garlanda, C.; Kennedy, G. D.; Nukaya, M.; Mantovani, A.; Kopan, R.; Bradfield, C. A.; Newberry, R. D.; Colonna, M. AHR Drives the Development of Gut ILC22 Cells and Postnatal Lymphoid Tissues via Pathways Dependent on and Independent of Notch. Nat. Immunol. 2012, 13 (2), 144151.

(69) Muri, J.; Heer, S.; Matsushita, M.; Pohlmeier, L.; Tortola, L.; Fuhrer, T.; Conrad, M.; Zamboni, N.; Kisielow, J.; Kopf, M. The Thioredoxin-1 System Is Essential for Fueling DNA Synthesis during T-Cell Metabolic Reprogramming and Proliferation. Nat. Commun. 2018, 9 (1), 1851. 